\title{
Vertebral and Intraspinal Anomalies in Indian Population with Congenital Scoliosis: A Study of 119 Consecutive Patients
}

\author{
Neeraj Gupta, Rajasekaran S, Balamurali G, Ajoy Shetty \\ Department of Orthopaedics and Spine Surgery, Ganga Hospital, Coimbatore, India
}

Study Design: Retrospective case study by clinical and radiological data analysis.

Purpose: To analyze different types of vertebral anomalies and the incidence of associated intraspinal anomalies in the Indian population. Overview of Literature: This is the largest study of congenital scoliosis and associated intraspinal anomalies in Indian population. Incidence of intraspinal anomaly in this series is $47 \%$ which is higher than previous literature. Hemivertebra was the most common anomaly as seen in previous studies.

Methods: A total of 119 patients with congenital scoliosis who underwent surgery between December 2006 and December 2012 were studied. Data was reviewed with medical records, plain radiographs, and magnetic resonance imaging (MRI) scans.

Results: Thoracolumbar curve was most common, seen in $43.6 \%$ of patients. In addition to scoliotic deformity, kyphosis was seen in $26 \%$ of patients. Failure of formation, the most common vertebral anomaly, was seen in $51.2 \%$ of patients, failure of segmentation was seen in $19.3 \%$ of patients, and there were $29.4 \%$ patients having both formation and segmentation anomalies. Hemivertebra was the most common vertebral anomaly seen in $66.3 \%$ of patients and for whom $63.2 \%$ were in thoracic spine. Intraspinal anomalies were associated with $47 \%$ of patients with congenital scoliosis. Tethered cord was the most common intraspinal abnormality and was found in $48.2 \%$ patients with intraspinal anomalies. The patients with failure of segmentation and mixed deformities were found to have a significantly higher incidence of intraspinal anomalies ( $65 \%$ and $57 \%$, respectively) than those with failure of formation (34\%). Out of 31 patients with kyphotic deformity 29\% had intraspinal anomalies, and amongst them tethered cord was the most common anomaly seen in $66 \%$ patients. Out of 12 patients with neurocutaneous markers, $83 \%$ patients had intraspinal anomaly.

Conclusions: Intraspinal anomalies were seen in $47 \%$ of patients with congenital scoliosis in the Indian population. Tethered cord was the most common intraspinal anomaly, seen in $48 \%$ cases of congenital scoliosis. Hemivertebra was seen in $66 \%$ cases and was the most common vertebral defect. Hence MRI imaging of whole spine should be done in all cases of congenital scoliosis before any surgical intervention. Special attention should be given to physical examination and visualization of any neurocutaneous markers, which are associated with a higher incidence of intraspinal anomalies; absence of a neurocutaneous marker, however, does not rule out intraspinal anomaly.

Keywords: Congenital scoliosis; Intraspinal anomalies; Tethered cord; Hemivertebra; Deformity

Received Jul 08, 2015; Revised Jul 21, 2015; Accepted Jul 21, 2015

Corresponding author: Neeraj Gupta

Department of Orthopaedics and Spine Surgery, Ganga Hospital , Coimbatore ,India

E-mail: neerajgupta691@gmail.com 


\section{Introduction}

Congenital scoliosis is the presence of abnormal coronal plane curvature in spine secondary to an anomalous congenital vertebral defect. It occurs 1 in 1,000 newborns $[1,2]$ and is much less common than idiopathic scoliosis. Vertebral anomalies can produce imbalance in the longitudinal growth of spine and lead to progressive congenital scoliosis [3,4]. Being a developmental anomaly, it is associated with intraspinal, genitourinary, cardiovascular, and other general abnormalities $[4,5]$. Congenital scoliosis is often associated with intraspinal anomalies. The embryonic development of vertebrae is closely related with that of spinal cord and it is not surprising that neural and vertebral malformations often co-exist [5]. These intraspinal anomalies include tethering of the cord, diastematomyelia, lipomas, syringomyelia and lipomeningocele. McMaster [5] reported intraspinal anomaly in $18 \%$ of 251 patients with myelography. Magnetic resonance imaging (MRI) is noninvasive and more sensitive in detecting the intraspinal abnormalities. Using MRI, Bradford et al. [6] reported that $38 \%$ of the 42 patients in their study had an intraspinal anomaly. Liu et al. [7] evaluated 539 Chinese patients with congenital scoliosis using MRI and found intraspinal anomalies in $24.5 \%$ of the patients and were more common in females. Out of 539 patients, $70 \%$ patients had failure of formation of vertebrae, $31.2 \%$ had failure of segmentation, and $13.2 \%$ had mixed abnormalities. There exists a high incidence of intraspinal anomalies in patients with congenital scoliosis but since the overlying skin is normal and associated neural abnormalities are mild or absent [5], they are sometimes overlooked. And in these patients, the normal movement of spinal cord in the spinal canal may be restricted, and any attempt to correct scoliosis could result in stretching of spinal cord and serious neurological complications. Therefore, before correcting scoliosis, it is essential to detect any intraspinal anomaly. There has been no longterm study in the Indian population to study the incidence of various vertebral anomalies and associated intraspinal anomalies in congenital scoliosis. In the present study, our aim was to study and analyze different types of vertebral anomalies and the incidence of associated intraspinal anomalies in 119 consecutive cases of congenital scoliosis in the Indian population at a single center.

\section{Materials and Methods}

A total of 119 patients with congenital scoliosis underwent surgical treatment between December 2006 and December 2012. A sequential study was done to analyze the different types of structural vertebral anomalies and the incidence of various intraspinal anomalies in congenital scoliosis patients in the Indian population. Institutional Review Board approval was obtained before the study. All patients underwent a full clinical examination, plain radiograph of the entire spine and neural axis MRI of whole spine including the posterior cranial fossa to screen the intraspinal and craniovertebral junction abnormities. Evaluation of the clinical findings, neurological examination and treatment, which were documented in medical records, was performed. Plain X-rays and MRI of all the patients were analyzed using Picture Archiving and Communication System. The congenital scoliosis was classified as resulting from the failure of formation, failure of segmentation, and mixed abnormalities. MRIs were studied by an experienced radiologist for any abnormalities in the hind brain, brain stem, spinal cord, nerve roots and covering membranes. Arnold Chiari-I malformation was considered when the tonsillar herniation was $5 \mathrm{~mm}$ or below the level of foramen magnum. The presence and type of anomaly in MRI was correlated to patient symptoms, clinical signs, and curve characteristics.

\section{Inclusion criteria}

All the patients with congenital scoliosis with clinical and radiological findings, with or without intraspinal anomalies in the above study period were included in the study.

\section{Exclusion criteria}

Patients with adolescent idiopathic scoliosis, pure kyphosis, adult onset scoliosis, scoliosis secondary to trauma, infection, tumor, meningocele, meningomyelocele, and neurofibromatosis were excluded from the study. Different types of structural bony abnormalities in congenital scoliosis, and incidence of intraspinal anomalies in them were analyzed. 


\section{Results}

\section{Demographics}

In the present study, out of 119 cases operated for congenital scoliosis, 44 were males (36.9\%) and 75 were females (63\%), depicting higher incidence of congenital scoliosis in females. Patient age at the time when the curve was noticed ranged from younger than 1 to 26 years with a mean of about 5.3 years.

Deformity curve distribution and vertebral anomalies (Table 1).

Thoracolumbar curve was most common and was seen in $43.6 \%$ of patients (52/119). In addition to scoliotic deformity, kyphosis was seen in $26 \%$ of patients (31/119). Failure of formation was the most common vertebral anomaly seen in $51.2 \%$ of patients (61/119). Failure of segmentation was seen in $19.3 \%$ of patients (23/119), and there were $29.4 \%$ of patients (35/119) having both formation and segmentation anomalies. Of the 61 patients with failure of formation, $60.6 \%$ (37/61) were in thoracic, $31.1 \%(19 / 61)$ in lumbar, $4.9 \%(3 / 61)$ in thoracolumbar, and $3.2 \%(2 / 61)$ were in sacral region. Of the 23 patients with failure of segmentation, $65.2 \%(15 / 23)$ were in thoracic, $4.3 \%(1 / 23)$ in cervical, $4.3 \%(1 / 23)$ in cervicothoracic, $13 \%(3 / 23)$ in thoracolumbar, and 13\% (3/23) were in lumbar region. Out of the 35 patients having mixed defects (both formation and segmentation defects), 65.7\% $(23 / 35)$ were in thoracic, $22.8 \%(8 / 35)$ in thoracolumbar, $8.5 \%(3 / 35)$ in lumbar region, and $2.8 \%(1 / 35)$ were in cervicothoracic region. Hemivertebra was the most common vertebral anomaly seen in congenital scoliosis. Out of 119 patients, 79 (66.3\%) had hemivertebra out of which $63.2 \%(50 / 79)$ were in thoracic spine, 30.3\% (24/79) were in lumbar spine, $2.5 \%(2 / 79)$ were in sacral region, and $3.7 \%(3 / 79)$ were in multiple locations.

\section{Intraspinal anomalies}

Of all the patients included in the study, 56 patients (47\%) were found to have intraspinal anomalies (Table 2). Females had significantly higher incidence of intraspinal anomalies than males, as there were $71.4 \%$ females (40/56) and $28.5 \%$ males (16/56) with intraspinal anomalies. $35.7 \%$ of patients (20/56) had multiple intraspinal abnormalities. Tethered cord was the most common intraspinal abnormality and was found in $48.2 \%$ of patients (27/56). Next most common intraspinal anomaly was diastematomyelia seen in $32.1 \%$ patients $(18 / 56)$. Out of these, $11 \%(2 / 18)$ were males and $89 \%$ females (16/18). Thirty-three percent patients (6/18) were of Type I and 67\% (12/18) patients were of Type II diastematomyelia. Syringomyelia was seen in $30.3 \%$ of patients $(17 / 56)$ with intraspinal anomalies. Syringomyelia alone was seen in $17.8 \%(10 / 56)$ and was associated with tethered cord in $8.9 \%(5 / 56)$ and diastematomyelia in $3.5 \%$ of cases $(2 / 56)$.

Intraspinal anomalies and vertebral anomalies analysis (Table 3).

Intraspinal anomalies were seen in $34.4 \%$ of patients $(21 / 61)$ with failure of formation, $65.2 \%$ of patients (15/23) with failure of segmentation, and $57.1 \%$ of the patients (20/35) with mixed deformity. The patients with failure of segmentation had higher incidence of intraspinal

Table 2. Intraspinal anomalies

\begin{tabular}{llc}
$\begin{array}{l}\text { Serial } \\
\text { no. }\end{array}$ & \multicolumn{1}{c}{ Intraspinal anomaly } & $\begin{array}{c}\text { No. of patients } \\
(56 / 119)\end{array}$ \\
\hline 1 & Tethered cord and low conus & $27(48.2)$ \\
\hline 2 & Diastematomyelia & $18(32.1)$ \\
\hline 3 & Syringomyelia & $17(30.3)$ \\
\hline 4 & Arnold Chiari malformation & $11(19.6)$ \\
\hline 5 & Intradural cyst & $1(1.7)$ \\
6 & Intradural lipoma & $2(3.5)$ \\
\hline
\end{tabular}

Values are presented as percent (number).

$35.7 \%$ patients (20/56) had multiple intraspinal abnormalities.

Table 1. Vertebral anomaly distribution

\begin{tabular}{|c|c|c|c|c|c|c|c|}
\hline Vertebral anomaly & Number & Cervical & Cervicothoracic & Thoracic & Lumbar & Thoracolumbar & Sacral \\
\hline Failure of formation & $51.2(61 / 119)$ & - & - & $60.6(37 / 61)$ & $31.1(19 / 61)$ & $4.9(3 / 61)$ & $3.2(2 / 61)$ \\
\hline Failure of segmentation & $19.3(23 / 119)$ & $4.3(1 / 23)$ & $13(1 / 23)$ & $65.2(15 / 23)$ & $13(3 / 23)$ & $13(3 / 23)$ & - \\
\hline Mixed & $29.4(35 / 119)$ & - & $2.8(1 / 35)$ & $65.7(23 / 35)$ & $8.5(3 / 35)$ & $22.8(8 / 35)$ & - \\
\hline
\end{tabular}

Values are presented as percent (number). 
Table 3. Vertebral and intraspinal anomalies

\begin{tabular}{lcccr} 
Vertebral anomaly & Intraspinal anomalies & Tethered cord & Diastematomyelia & Syringomyelia \\
Failure of formation & $34(21 / 61)$ & $47.6(10 / 21)$ & $23.8(5 / 21)$ & $38(8 / 21)$ \\
Failure of segmentation & $65.2(15 / 23)$ & $60(9 / 15)$ & $40(6 / 15)$ & $13.3(2 / 15)$ \\
\hline Mixed & $57.1(20 / 35)$ & $40(8 / 20)$ & $35(7 / 20)$ & $35(5 / 20)$ \\
\hline
\end{tabular}

Values are presented as percent (number).

$35.7 \%$ patients $(20 / 56)$ had multiple intraspinal abnormalitie.

Table 4. Neurocutaneous markers

\begin{tabular}{llc}
$\begin{array}{l}\text { Serial } \\
\text { no. }\end{array}$ & Neurocutaneous marker & $\begin{array}{c}\text { No. of patients } \\
(12 / 119)\end{array}$ \\
1 & Tuft of hair & $5^{\text {a) }}+1=6$ \\
2 & Dermoid cyst & $1^{\text {a) }}$ \\
\hline 3 & Nevus & $2^{\text {a) }}$ \\
\hline 4 & Dimple & $1^{\text {a) }}$ \\
\hline 6 & Hypopigmented macule & 1 \\
\hline
\end{tabular}

a)Intraspinal anomaly present.

anomalies than patients with mixed deformities, which was higher than those with failure of formation. Out of the 21 patients with intraspinal anomalies with failure of formation, tethered cord was seen in $47.6 \%$ of patients $(10 / 21)$, followed by syringomyelia in $38 \%(8 / 21)$ and diastematomyelia in $23.8 \%$ of patients $(5 / 21)$. Out of the 15 patients of intraspinal anomalies with failure of segmentation, tethered cord was seen in $60 \%$ of the cases (9/15), followed by diastematomyelia in $40 \%$ (6/15), and syringomyelia in $13.3 \%$ of the cases (2/15). Syringomyelia was associated with diastematomyelia in both the cases. Out of the 20 patients with intraspinal anomalies with mixed deformities, tethered cord was seen in $40 \%(8 / 20)$, followed by diastematomyelia in $35 \%$ of the cases $(7 / 20)$ and syringomyelia in $25 \%(5 / 20)$. Out of 31 patients with kyphotic deformity, 29\% (9/31) had intraspinal anomalies, and amongst them tethered cord was the most common anomaly seen in $66 \%$ of patients (6/9).

\section{Physical findings}

Out of all the cases (119) studied, 17 cases (14.2\%) presented with pain, 11 cases (9.2\%) with limb weakness, 7 cases (5.8\%) with sensory disturbances, 23 cases (19.3\%) with difficulty in walking, 11 cases $(9.2 \%)$ with headache and 18 cases (15.1\%) with bowel bladder disturbances. Neurological examination at the time of presentation revealed motor deficits in 13 cases (10.9\%), sensory loss in 4 cases $(3.3 \%)$, asymmetric abdominal reflexes in 2 cases (1.6\%), increased deep tendon reflexes in 14 cases (11.7\%) and Babinski positivity in 12 cases (10\%). Neural deficits were seen almost equally in patients, with or without intraspinal anomaly. Neurocutaneous markers (Table 4) were seen more commonly in patients with intraspinal anomalies. Out of 12 patients with neurocutaneous markers, $83 \%$ patients $(10 / 12)$ had intraspinal anomaly.

\section{Discussion}

To our knowledge, this is the largest study of congenital scoliosis and analysis of associated intraspinal anomalies in the Indian population. Average age at which a patient presented with deformity was similar to McMaster [5] series. Female preponderance of congenital scoliosis and intraspinal anomalies was similar to all the previous reports [5,7-9]. Thoracolumbar curve was the most common deformity in our study, which was similar to the study of Mohanty and Kumar [10], but it differed from observation of McMaster and Ohtsuka [3] in which there was a preponderance of lower thoracic curve. In our study, there were $51.2 \%$ cases (61/119) of failure of formation, 19.3\% cases (23/119) of failure of segmentation, and $29.4 \%$ cases (35/119) with mixed defects. There were no significant differences between our observation and in the study by Basu et al. [11] in which there were 53\% (67/126) cases of failure of formation, $8 \%(10 / 126)$ of failure of segmentation, and $26 \%(33 / 126)$ of mixed defects. In the study by Liu et al. [7] the failure of formation was seen in $70 \%$ (361/539) of cases with failure of segmentation in 31.2\% $(168 / 539)$ and mixed deformities in 13\% (70/539) of cases. Hemivertebra was the most common vertebral anomaly as seen in previous studies [7-11].

Several studies have revealed the association of intra- 
spinal anomaly with congenital spine deformity. In the present study, the incidence of intraspinal anomaly was $47 \%$, which is higher than reported in previous literature. McMaster [5] reported incidence of $18.3 \%$ in his series, in which 106 of 251 underwent myelography, and diastematomyelia was diagnosed in eight patients on the basis of midline spur in plain radiograph. Because of toxic effects of oil-based contrast, myelography was performed only in patients with neurological signs and before surgical correction. The lower incidence seen may be because myelography was not done in all patients. Intraspinal anomalies in Bradford et al. [6] and Erfani et al. [8] series was $38 \%(n=16)$ and $41.3 \%(n=19)$ in which 42 and 46 patients with congenital scoliosis were respectively studied with MRI correlation, which is lower as compared to $47 \%$ in our study. This difference may be because the number of cases in Bradford and Erfani series were fewer. Tethered cord was the most common intraspinal anomaly seen, as also seen in previous studies [6,9,11-13]. Diastematomyelia was the most common intraspinal anomaly in McMaster series [5]. Female preponderance of diastematomyelia was similar to previous literature $[4,14]$ but the percentage of Type I diastematomyelia was less as compared to study by Cheng et al. [14]. This may be due to less number of cases with diastematomyelia in our study.

The incidence of intraspinal anomaly in patients with failures of segmentation and mixed defects were significantly higher than patients with failures of formation in this study, which was in line with studies by Basu et al. [11] and McMaster [5]. Intraspinal anomalies were seen in $34 \%$ of patients with failure of formation, $65.2 \%$ with failure of segmentation, and $57 \%$ with mixed deformity. In the study by Basu et al. [11] intraspinal anomalies were $30 \%$ in failure of formation, $40 \%$ in failure of segmentation, and $40 \%$ in mixed deformity. There were no significant differences between the results. Thoracic spine was the most common site for vertebral malformations, either due to failure of formation, failure of segmentation or in mixed defects. There was no significant statistical difference between results of neurological examination between patients with or without intraspinal anomalies. In our study, $83 \%$ of patients with neurocutaneous markers had intraspinal anomaly. This was in contradiction to the studies by McMaster [5] and Bradford et al. [6] who found neurocutaneous stigmas as unreliable indicators of intraspinal anomalies.

\section{Conclusions}

Intraspinal anomalies were associated with $47 \%$ of patients with congenital scoliosis in the Indian population. $71 \%$ of congenital scoliosis patients with intraspinal anomalies were females. Hemivertebra was the most common vertebral anomaly in congenital scoliosis, and it was most commonly located in thoracic spine. Intraspinal anomaly was most common in vertebrae with segmentation and mixed defects. Tethered cord was the most common intraspinal anomaly in the Indian population. Special attention should be given to physical examination and inspecting for any neurocutaneous markers, which are associated with high incidence of intraspinal anomalies; however, absence of neurocutaneous marker does not rule out intraspinal anomaly. MRI should be the part of routine protocol for assessment of congenital scoliosis patients before contemplating any surgical procedure.

\section{Conflict of Interest}

No potential conflict of interest relevant to this article was reported.

\section{References}

1. Shands AR Jr, Bundens WD. Congenital deformities of the spine; an analysis of the roentgenograms of 700 children. Bull Hosp Joint Dis 1956;17:110-33.

2. Giampietro PF, Blank RD, Raggio CL, et al. Congenital and idiopathic scoliosis: clinical and genetic aspects. Clin Med Res 2003;1:125-36.

3. McMaster MJ, Ohtsuka K. The natural history of congenital scoliosis: a study of two hundred and fiftyone patients. J Bone Joint Surg Am 1982;64:1128-47.

4. Winter RB, Moe JH, Eilers VE. Congenital scoliosis a study of 234 patients treated and untreated. Part I: natural history. J Bone Joint Surg Am 1968;50:1-15.

5. McMaster MJ. Occult intraspinal anomalies and congenital scoliosis. J Bone Joint Surg Am 1984;66:588601.

6. Bradford DS, Heithoff KB, Cohen M. Intraspinal abnormalities and congenital spine deformities: a radiographic and MRI study. J Pediatr Orthop 1991;11:3641.

7. Liu YT, Guo LL, Tian Z, et al. A retrospective study of congenital scoliosis and associated cardiac and 
intraspinal abnormities in a Chinese population. Eur Spine J 2011;20:2111-4.

8. Erfani MA, Ganjavian MS, Ameri E, Namazi H, Solooki S. Occult intraspinal abnormalities and congenital scoliosis. J Res Med Sci 2007;12:53-7.

9. Rajasekaran S, Kamath V, Kiran R, Shetty AP. Intraspinal anomalies in scoliosis: an MRI analysis of 177 consecutive scoliosis patients. Indian J Orthop 2010;44:57-63.

10. Mohanty S, Kumar N. Patterns of presentation of congenital scoliosis. J Orthop Surg (Hong Kong) 2000;8:33-37.

11. Basu PS, Elsebaie H, Noordeen MH. Congenital spinal deformity: a comprehensive assessment at presentation. Spine (Phila Pa 1976) 2002;27:2255-9.

12. Suh SW, Sarwark JF, Vora A, Huang BK. Evaluating congenital spine deformities for intraspinal anomalies with magnetic resonance imaging. J Pediatr Orthop 2001;21:525-31.

13. Prahinski JR, Polly DW Jr, McHale KA, Ellenbogen RG. Occult intraspinal anomalies in congenital scoliosis. J Pediatr Orthop 2000;20:59-63.

14. Cheng B, Li FT, Lin L. Diastematomyelia: a retrospective review of 138 patients. J Bone Joint Surg Br 2012;94:365-72. 